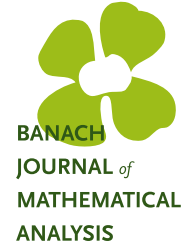

Banach J. Math. Anal. 10 (2016), no. 3, 566-592

http://dx.doi.org/10.1215/17358787-3607354

ISSN: $1735-8787$ (electronic)

http://projecteuclid.org/bjma

\title{
INTRINSIC ATOMIC AND MOLECULAR DECOMPOSITIONS OF HARDY-MUSIELAK-ORLICZ SPACES
}

\author{
KWOK-PUN HO
}

Communicated by T. Sugawa

\begin{abstract}
We introduce the Hardy type space for Musielak-Orlicz spaces. It includes several existing Hardy type spaces such as the Hardy-Orlicz spaces and the Hardy spaces with variable exponents. Furthermore, we develop an atomic decomposition such that the size condition just relies on the norms of Musielak-Orlicz spaces. This gives us a nature extension of the molecular decompositions to the Hardy type space for Musielak-Orlicz spaces.
\end{abstract}

\section{INTRODUCTION}

In this article we aim to establish the atomic and molecular decompositions for the Hardy spaces built on the Musielak-Orlicz spaces.

Once Fefferman, Stein, and Weiss introduced the classical Hardy spaces $H^{p}$, they became one of the most important function spaces in analysis. Recently, there have been several extensions of classical Hardy spaces. We have the Hardy-Orlicz spaces which are the Orlicz space version of the classical Hardy spaces (see [18], [17], [28], [35]). The Hardy spaces with variable exponents were introduced and studied in [27] and [30]. The atomic decomposition for weighted Hardy spaces with variable exponents is developed in [14].

We also have the atomic decompositions for the Hardy-Morrey spaces (see [11], [16]). The reader may also consult [31] and [37] for some detailed studies on the Triebel-Lizorkin-Morrey spaces which are generalizations of the Hardy-Morrey spaces.

Copyright 2016 by the Tusi Mathematical Research Group.

Received Aug. 24, 2015; Accepted Nov. 11, 2015.

2010 Mathematics Subject Classification. Primary 42B30; Secondary 42B25, 42B35, 46E30.

Keywords. atomic decomposition, molecular decomposition, Hardy spaces, Musielak-Orlicz spaces. 


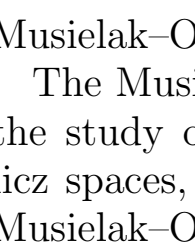

Banach J. Math. Anal. 10 (2016), no. 3, 566-592

http://dx.doi.org/10.1215/17358787-3607354

ISSN: $1735-8787$ (electronic)

http://projecteuclid.org/bjma

\title{
INTRINSIC ATOMIC AND MOLECULAR DECOMPOSITIONS OF HARDY-MUSIELAK-ORLICZ SPACES
}

\author{
KWOK-PUN HO
}

Communicated by T. Sugawa

\begin{abstract}
We introduce the Hardy type space for Musielak-Orlicz spaces. It includes several existing Hardy type spaces such as the Hardy-Orlicz spaces and the Hardy spaces with variable exponents. Furthermore, we develop an atomic decomposition such that the size condition just relies on the norms of Musielak-Orlicz spaces. This gives us a nature extension of the molecular decompositions to the Hardy type space for Musielak-Orlicz spaces.
\end{abstract}

\section{INTRODUCTION}

In this article we aim to establish the atomic and molecular decompositions for the Hardy spaces built on the Musielak-Orlicz spaces.

Once Fefferman, Stein, and Weiss introduced the classical Hardy spaces $H^{p}$, they became one of the most important function spaces in analysis. Recently, there have been several extensions of classical Hardy spaces. We have the Hardy-Orlicz spaces which are the Orlicz space version of the classical Hardy spaces (see [18], [17], [28], [35]). The Hardy spaces with variable exponents were introduced and studied in [27] and [30]. The atomic decomposition for weighted Hardy spaces with variable exponents is developed in [14].

We also have the atomic decompositions for the Hardy-Morrey spaces (see [11], [16]). The reader may also consult [31] and [37] for some detailed studies on the Triebel-Lizorkin-Morrey spaces which are generalizations of the Hardy-Morrey spaces.

Copyright 2016 by the Tusi Mathematical Research Group.

Received Aug. 24, 2015; Accepted Nov. 11, 2015.

2010 Mathematics Subject Classification. Primary 42B30; Secondary 42B25, 42B35, 46E30.

Keywords. atomic decomposition, molecular decomposition, Hardy spaces, Musielak-Orlicz spaces. 


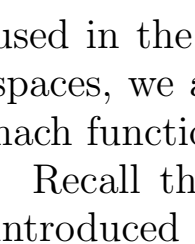

Banach J. Math. Anal. 10 (2016), no. 3, 566-592

http://dx.doi.org/10.1215/17358787-3607354

ISSN: $1735-8787$ (electronic)

http://projecteuclid.org/bjma

\title{
INTRINSIC ATOMIC AND MOLECULAR DECOMPOSITIONS OF HARDY-MUSIELAK-ORLICZ SPACES
}

\author{
KWOK-PUN HO
}

Communicated by T. Sugawa

\begin{abstract}
We introduce the Hardy type space for Musielak-Orlicz spaces. It includes several existing Hardy type spaces such as the Hardy-Orlicz spaces and the Hardy spaces with variable exponents. Furthermore, we develop an atomic decomposition such that the size condition just relies on the norms of Musielak-Orlicz spaces. This gives us a nature extension of the molecular decompositions to the Hardy type space for Musielak-Orlicz spaces.
\end{abstract}

\section{INTRODUCTION}

In this article we aim to establish the atomic and molecular decompositions for the Hardy spaces built on the Musielak-Orlicz spaces.

Once Fefferman, Stein, and Weiss introduced the classical Hardy spaces $H^{p}$, they became one of the most important function spaces in analysis. Recently, there have been several extensions of classical Hardy spaces. We have the Hardy-Orlicz spaces which are the Orlicz space version of the classical Hardy spaces (see [18], [17], [28], [35]). The Hardy spaces with variable exponents were introduced and studied in [27] and [30]. The atomic decomposition for weighted Hardy spaces with variable exponents is developed in [14].

We also have the atomic decompositions for the Hardy-Morrey spaces (see [11], [16]). The reader may also consult [31] and [37] for some detailed studies on the Triebel-Lizorkin-Morrey spaces which are generalizations of the Hardy-Morrey spaces.

Copyright 2016 by the Tusi Mathematical Research Group.

Received Aug. 24, 2015; Accepted Nov. 11, 2015.

2010 Mathematics Subject Classification. Primary 42B30; Secondary 42B25, 42B35, 46E30.

Keywords. atomic decomposition, molecular decomposition, Hardy spaces, Musielak-Orlicz spaces. 


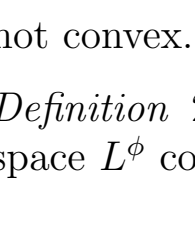

Banach J. Math. Anal. 10 (2016), no. 3, 566-592

http://dx.doi.org/10.1215/17358787-3607354

ISSN: $1735-8787$ (electronic)

http://projecteuclid.org/bjma

\title{
INTRINSIC ATOMIC AND MOLECULAR DECOMPOSITIONS OF HARDY-MUSIELAK-ORLICZ SPACES
}

\author{
KWOK-PUN HO
}

Communicated by T. Sugawa

\begin{abstract}
We introduce the Hardy type space for Musielak-Orlicz spaces. It includes several existing Hardy type spaces such as the Hardy-Orlicz spaces and the Hardy spaces with variable exponents. Furthermore, we develop an atomic decomposition such that the size condition just relies on the norms of Musielak-Orlicz spaces. This gives us a nature extension of the molecular decompositions to the Hardy type space for Musielak-Orlicz spaces.
\end{abstract}

\section{INTRODUCTION}

In this article we aim to establish the atomic and molecular decompositions for the Hardy spaces built on the Musielak-Orlicz spaces.

Once Fefferman, Stein, and Weiss introduced the classical Hardy spaces $H^{p}$, they became one of the most important function spaces in analysis. Recently, there have been several extensions of classical Hardy spaces. We have the Hardy-Orlicz spaces which are the Orlicz space version of the classical Hardy spaces (see [18], [17], [28], [35]). The Hardy spaces with variable exponents were introduced and studied in [27] and [30]. The atomic decomposition for weighted Hardy spaces with variable exponents is developed in [14].

We also have the atomic decompositions for the Hardy-Morrey spaces (see [11], [16]). The reader may also consult [31] and [37] for some detailed studies on the Triebel-Lizorkin-Morrey spaces which are generalizations of the Hardy-Morrey spaces.

Copyright 2016 by the Tusi Mathematical Research Group.

Received Aug. 24, 2015; Accepted Nov. 11, 2015.

2010 Mathematics Subject Classification. Primary 42B30; Secondary 42B25, 42B35, 46E30.

Keywords. atomic decomposition, molecular decomposition, Hardy spaces, Musielak-Orlicz spaces. 


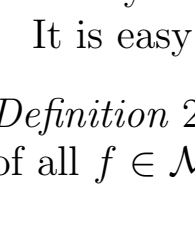

Banach J. Math. Anal. 10 (2016), no. 3, 566-592

http://dx.doi.org/10.1215/17358787-3607354

ISSN: $1735-8787$ (electronic)

http://projecteuclid.org/bjma

\title{
INTRINSIC ATOMIC AND MOLECULAR DECOMPOSITIONS OF HARDY-MUSIELAK-ORLICZ SPACES
}

\author{
KWOK-PUN HO
}

Communicated by T. Sugawa

\begin{abstract}
We introduce the Hardy type space for Musielak-Orlicz spaces. It includes several existing Hardy type spaces such as the Hardy-Orlicz spaces and the Hardy spaces with variable exponents. Furthermore, we develop an atomic decomposition such that the size condition just relies on the norms of Musielak-Orlicz spaces. This gives us a nature extension of the molecular decompositions to the Hardy type space for Musielak-Orlicz spaces.
\end{abstract}

\section{INTRODUCTION}

In this article we aim to establish the atomic and molecular decompositions for the Hardy spaces built on the Musielak-Orlicz spaces.

Once Fefferman, Stein, and Weiss introduced the classical Hardy spaces $H^{p}$, they became one of the most important function spaces in analysis. Recently, there have been several extensions of classical Hardy spaces. We have the Hardy-Orlicz spaces which are the Orlicz space version of the classical Hardy spaces (see [18], [17], [28], [35]). The Hardy spaces with variable exponents were introduced and studied in [27] and [30]. The atomic decomposition for weighted Hardy spaces with variable exponents is developed in [14].

We also have the atomic decompositions for the Hardy-Morrey spaces (see [11], [16]). The reader may also consult [31] and [37] for some detailed studies on the Triebel-Lizorkin-Morrey spaces which are generalizations of the Hardy-Morrey spaces.

Copyright 2016 by the Tusi Mathematical Research Group.

Received Aug. 24, 2015; Accepted Nov. 11, 2015.

2010 Mathematics Subject Classification. Primary 42B30; Secondary 42B25, 42B35, 46E30.

Keywords. atomic decomposition, molecular decomposition, Hardy spaces, Musielak-Orlicz spaces. 


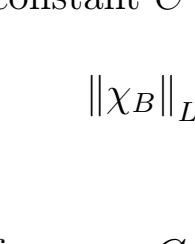

Banach J. Math. Anal. 10 (2016), no. 3, 566-592

http://dx.doi.org/10.1215/17358787-3607354

ISSN: $1735-8787$ (electronic)

http://projecteuclid.org/bjma

\title{
INTRINSIC ATOMIC AND MOLECULAR DECOMPOSITIONS OF HARDY-MUSIELAK-ORLICZ SPACES
}

\author{
KWOK-PUN HO
}

Communicated by T. Sugawa

\begin{abstract}
We introduce the Hardy type space for Musielak-Orlicz spaces. It includes several existing Hardy type spaces such as the Hardy-Orlicz spaces and the Hardy spaces with variable exponents. Furthermore, we develop an atomic decomposition such that the size condition just relies on the norms of Musielak-Orlicz spaces. This gives us a nature extension of the molecular decompositions to the Hardy type space for Musielak-Orlicz spaces.
\end{abstract}

\section{INTRODUCTION}

In this article we aim to establish the atomic and molecular decompositions for the Hardy spaces built on the Musielak-Orlicz spaces.

Once Fefferman, Stein, and Weiss introduced the classical Hardy spaces $H^{p}$, they became one of the most important function spaces in analysis. Recently, there have been several extensions of classical Hardy spaces. We have the Hardy-Orlicz spaces which are the Orlicz space version of the classical Hardy spaces (see [18], [17], [28], [35]). The Hardy spaces with variable exponents were introduced and studied in [27] and [30]. The atomic decomposition for weighted Hardy spaces with variable exponents is developed in [14].

We also have the atomic decompositions for the Hardy-Morrey spaces (see [11], [16]). The reader may also consult [31] and [37] for some detailed studies on the Triebel-Lizorkin-Morrey spaces which are generalizations of the Hardy-Morrey spaces.

Copyright 2016 by the Tusi Mathematical Research Group.

Received Aug. 24, 2015; Accepted Nov. 11, 2015.

2010 Mathematics Subject Classification. Primary 42B30; Secondary 42B25, 42B35, 46E30.

Keywords. atomic decomposition, molecular decomposition, Hardy spaces, Musielak-Orlicz spaces. 


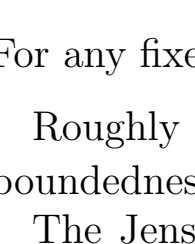

Banach J. Math. Anal. 10 (2016), no. 3, 566-592

http://dx.doi.org/10.1215/17358787-3607354

ISSN: $1735-8787$ (electronic)

http://projecteuclid.org/bjma

\title{
INTRINSIC ATOMIC AND MOLECULAR DECOMPOSITIONS OF HARDY-MUSIELAK-ORLICZ SPACES
}

\author{
KWOK-PUN HO
}

Communicated by T. Sugawa

\begin{abstract}
We introduce the Hardy type space for Musielak-Orlicz spaces. It includes several existing Hardy type spaces such as the Hardy-Orlicz spaces and the Hardy spaces with variable exponents. Furthermore, we develop an atomic decomposition such that the size condition just relies on the norms of Musielak-Orlicz spaces. This gives us a nature extension of the molecular decompositions to the Hardy type space for Musielak-Orlicz spaces.
\end{abstract}

\section{INTRODUCTION}

In this article we aim to establish the atomic and molecular decompositions for the Hardy spaces built on the Musielak-Orlicz spaces.

Once Fefferman, Stein, and Weiss introduced the classical Hardy spaces $H^{p}$, they became one of the most important function spaces in analysis. Recently, there have been several extensions of classical Hardy spaces. We have the Hardy-Orlicz spaces which are the Orlicz space version of the classical Hardy spaces (see [18], [17], [28], [35]). The Hardy spaces with variable exponents were introduced and studied in [27] and [30]. The atomic decomposition for weighted Hardy spaces with variable exponents is developed in [14].

We also have the atomic decompositions for the Hardy-Morrey spaces (see [11], [16]). The reader may also consult [31] and [37] for some detailed studies on the Triebel-Lizorkin-Morrey spaces which are generalizations of the Hardy-Morrey spaces.

Copyright 2016 by the Tusi Mathematical Research Group.

Received Aug. 24, 2015; Accepted Nov. 11, 2015.

2010 Mathematics Subject Classification. Primary 42B30; Secondary 42B25, 42B35, 46E30.

Keywords. atomic decomposition, molecular decomposition, Hardy spaces, Musielak-Orlicz spaces. 


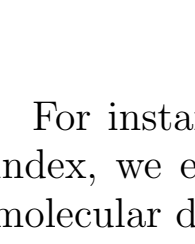

Banach J. Math. Anal. 10 (2016), no. 3, 566-592

http://dx.doi.org/10.1215/17358787-3607354

ISSN: $1735-8787$ (electronic)

http://projecteuclid.org/bjma

\title{
INTRINSIC ATOMIC AND MOLECULAR DECOMPOSITIONS OF HARDY-MUSIELAK-ORLICZ SPACES
}

\author{
KWOK-PUN HO
}

Communicated by T. Sugawa

\begin{abstract}
We introduce the Hardy type space for Musielak-Orlicz spaces. It includes several existing Hardy type spaces such as the Hardy-Orlicz spaces and the Hardy spaces with variable exponents. Furthermore, we develop an atomic decomposition such that the size condition just relies on the norms of Musielak-Orlicz spaces. This gives us a nature extension of the molecular decompositions to the Hardy type space for Musielak-Orlicz spaces.
\end{abstract}

\section{INTRODUCTION}

In this article we aim to establish the atomic and molecular decompositions for the Hardy spaces built on the Musielak-Orlicz spaces.

Once Fefferman, Stein, and Weiss introduced the classical Hardy spaces $H^{p}$, they became one of the most important function spaces in analysis. Recently, there have been several extensions of classical Hardy spaces. We have the Hardy-Orlicz spaces which are the Orlicz space version of the classical Hardy spaces (see [18], [17], [28], [35]). The Hardy spaces with variable exponents were introduced and studied in [27] and [30]. The atomic decomposition for weighted Hardy spaces with variable exponents is developed in [14].

We also have the atomic decompositions for the Hardy-Morrey spaces (see [11], [16]). The reader may also consult [31] and [37] for some detailed studies on the Triebel-Lizorkin-Morrey spaces which are generalizations of the Hardy-Morrey spaces.

Copyright 2016 by the Tusi Mathematical Research Group.

Received Aug. 24, 2015; Accepted Nov. 11, 2015.

2010 Mathematics Subject Classification. Primary 42B30; Secondary 42B25, 42B35, 46E30.

Keywords. atomic decomposition, molecular decomposition, Hardy spaces, Musielak-Orlicz spaces. 


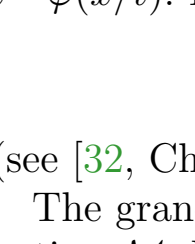

Banach J. Math. Anal. 10 (2016), no. 3, 566-592

http://dx.doi.org/10.1215/17358787-3607354

ISSN: $1735-8787$ (electronic)

http://projecteuclid.org/bjma

\title{
INTRINSIC ATOMIC AND MOLECULAR DECOMPOSITIONS OF HARDY-MUSIELAK-ORLICZ SPACES
}

\author{
KWOK-PUN HO
}

Communicated by T. Sugawa

\begin{abstract}
We introduce the Hardy type space for Musielak-Orlicz spaces. It includes several existing Hardy type spaces such as the Hardy-Orlicz spaces and the Hardy spaces with variable exponents. Furthermore, we develop an atomic decomposition such that the size condition just relies on the norms of Musielak-Orlicz spaces. This gives us a nature extension of the molecular decompositions to the Hardy type space for Musielak-Orlicz spaces.
\end{abstract}

\section{INTRODUCTION}

In this article we aim to establish the atomic and molecular decompositions for the Hardy spaces built on the Musielak-Orlicz spaces.

Once Fefferman, Stein, and Weiss introduced the classical Hardy spaces $H^{p}$, they became one of the most important function spaces in analysis. Recently, there have been several extensions of classical Hardy spaces. We have the Hardy-Orlicz spaces which are the Orlicz space version of the classical Hardy spaces (see [18], [17], [28], [35]). The Hardy spaces with variable exponents were introduced and studied in [27] and [30]. The atomic decomposition for weighted Hardy spaces with variable exponents is developed in [14].

We also have the atomic decompositions for the Hardy-Morrey spaces (see [11], [16]). The reader may also consult [31] and [37] for some detailed studies on the Triebel-Lizorkin-Morrey spaces which are generalizations of the Hardy-Morrey spaces.

Copyright 2016 by the Tusi Mathematical Research Group.

Received Aug. 24, 2015; Accepted Nov. 11, 2015.

2010 Mathematics Subject Classification. Primary 42B30; Secondary 42B25, 42B35, 46E30.

Keywords. atomic decomposition, molecular decomposition, Hardy spaces, Musielak-Orlicz spaces. 


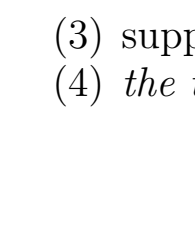

Banach J. Math. Anal. 10 (2016), no. 3, 566-592

http://dx.doi.org/10.1215/17358787-3607354

ISSN: $1735-8787$ (electronic)

http://projecteuclid.org/bjma

\title{
INTRINSIC ATOMIC AND MOLECULAR DECOMPOSITIONS OF HARDY-MUSIELAK-ORLICZ SPACES
}

\author{
KWOK-PUN HO
}

Communicated by T. Sugawa

\begin{abstract}
We introduce the Hardy type space for Musielak-Orlicz spaces. It includes several existing Hardy type spaces such as the Hardy-Orlicz spaces and the Hardy spaces with variable exponents. Furthermore, we develop an atomic decomposition such that the size condition just relies on the norms of Musielak-Orlicz spaces. This gives us a nature extension of the molecular decompositions to the Hardy type space for Musielak-Orlicz spaces.
\end{abstract}

\section{INTRODUCTION}

In this article we aim to establish the atomic and molecular decompositions for the Hardy spaces built on the Musielak-Orlicz spaces.

Once Fefferman, Stein, and Weiss introduced the classical Hardy spaces $H^{p}$, they became one of the most important function spaces in analysis. Recently, there have been several extensions of classical Hardy spaces. We have the Hardy-Orlicz spaces which are the Orlicz space version of the classical Hardy spaces (see [18], [17], [28], [35]). The Hardy spaces with variable exponents were introduced and studied in [27] and [30]. The atomic decomposition for weighted Hardy spaces with variable exponents is developed in [14].

We also have the atomic decompositions for the Hardy-Morrey spaces (see [11], [16]). The reader may also consult [31] and [37] for some detailed studies on the Triebel-Lizorkin-Morrey spaces which are generalizations of the Hardy-Morrey spaces.

Copyright 2016 by the Tusi Mathematical Research Group.

Received Aug. 24, 2015; Accepted Nov. 11, 2015.

2010 Mathematics Subject Classification. Primary 42B30; Secondary 42B25, 42B35, 46E30.

Keywords. atomic decomposition, molecular decomposition, Hardy spaces, Musielak-Orlicz spaces. 


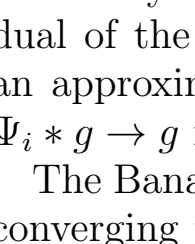

Banach J. Math. Anal. 10 (2016), no. 3, 566-592

http://dx.doi.org/10.1215/17358787-3607354

ISSN: $1735-8787$ (electronic)

http://projecteuclid.org/bjma

\title{
INTRINSIC ATOMIC AND MOLECULAR DECOMPOSITIONS OF HARDY-MUSIELAK-ORLICZ SPACES
}

\author{
KWOK-PUN HO
}

Communicated by T. Sugawa

\begin{abstract}
We introduce the Hardy type space for Musielak-Orlicz spaces. It includes several existing Hardy type spaces such as the Hardy-Orlicz spaces and the Hardy spaces with variable exponents. Furthermore, we develop an atomic decomposition such that the size condition just relies on the norms of Musielak-Orlicz spaces. This gives us a nature extension of the molecular decompositions to the Hardy type space for Musielak-Orlicz spaces.
\end{abstract}

\section{INTRODUCTION}

In this article we aim to establish the atomic and molecular decompositions for the Hardy spaces built on the Musielak-Orlicz spaces.

Once Fefferman, Stein, and Weiss introduced the classical Hardy spaces $H^{p}$, they became one of the most important function spaces in analysis. Recently, there have been several extensions of classical Hardy spaces. We have the Hardy-Orlicz spaces which are the Orlicz space version of the classical Hardy spaces (see [18], [17], [28], [35]). The Hardy spaces with variable exponents were introduced and studied in [27] and [30]. The atomic decomposition for weighted Hardy spaces with variable exponents is developed in [14].

We also have the atomic decompositions for the Hardy-Morrey spaces (see [11], [16]). The reader may also consult [31] and [37] for some detailed studies on the Triebel-Lizorkin-Morrey spaces which are generalizations of the Hardy-Morrey spaces.

Copyright 2016 by the Tusi Mathematical Research Group.

Received Aug. 24, 2015; Accepted Nov. 11, 2015.

2010 Mathematics Subject Classification. Primary 42B30; Secondary 42B25, 42B35, 46E30.

Keywords. atomic decomposition, molecular decomposition, Hardy spaces, Musielak-Orlicz spaces. 


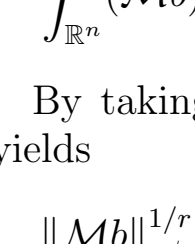

Banach J. Math. Anal. 10 (2016), no. 3, 566-592

http://dx.doi.org/10.1215/17358787-3607354

ISSN: $1735-8787$ (electronic)

http://projecteuclid.org/bjma

\title{
INTRINSIC ATOMIC AND MOLECULAR DECOMPOSITIONS OF HARDY-MUSIELAK-ORLICZ SPACES
}

\author{
KWOK-PUN HO
}

Communicated by T. Sugawa

\begin{abstract}
We introduce the Hardy type space for Musielak-Orlicz spaces. It includes several existing Hardy type spaces such as the Hardy-Orlicz spaces and the Hardy spaces with variable exponents. Furthermore, we develop an atomic decomposition such that the size condition just relies on the norms of Musielak-Orlicz spaces. This gives us a nature extension of the molecular decompositions to the Hardy type space for Musielak-Orlicz spaces.
\end{abstract}

\section{INTRODUCTION}

In this article we aim to establish the atomic and molecular decompositions for the Hardy spaces built on the Musielak-Orlicz spaces.

Once Fefferman, Stein, and Weiss introduced the classical Hardy spaces $H^{p}$, they became one of the most important function spaces in analysis. Recently, there have been several extensions of classical Hardy spaces. We have the Hardy-Orlicz spaces which are the Orlicz space version of the classical Hardy spaces (see [18], [17], [28], [35]). The Hardy spaces with variable exponents were introduced and studied in [27] and [30]. The atomic decomposition for weighted Hardy spaces with variable exponents is developed in [14].

We also have the atomic decompositions for the Hardy-Morrey spaces (see [11], [16]). The reader may also consult [31] and [37] for some detailed studies on the Triebel-Lizorkin-Morrey spaces which are generalizations of the Hardy-Morrey spaces.

Copyright 2016 by the Tusi Mathematical Research Group.

Received Aug. 24, 2015; Accepted Nov. 11, 2015.

2010 Mathematics Subject Classification. Primary 42B30; Secondary 42B25, 42B35, 46E30.

Keywords. atomic decomposition, molecular decomposition, Hardy spaces, Musielak-Orlicz spaces. 


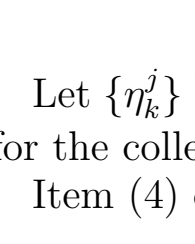

Banach J. Math. Anal. 10 (2016), no. 3, 566-592

http://dx.doi.org/10.1215/17358787-3607354

ISSN: $1735-8787$ (electronic)

http://projecteuclid.org/bjma

\title{
INTRINSIC ATOMIC AND MOLECULAR DECOMPOSITIONS OF HARDY-MUSIELAK-ORLICZ SPACES
}

\author{
KWOK-PUN HO
}

Communicated by T. Sugawa

\begin{abstract}
We introduce the Hardy type space for Musielak-Orlicz spaces. It includes several existing Hardy type spaces such as the Hardy-Orlicz spaces and the Hardy spaces with variable exponents. Furthermore, we develop an atomic decomposition such that the size condition just relies on the norms of Musielak-Orlicz spaces. This gives us a nature extension of the molecular decompositions to the Hardy type space for Musielak-Orlicz spaces.
\end{abstract}

\section{INTRODUCTION}

In this article we aim to establish the atomic and molecular decompositions for the Hardy spaces built on the Musielak-Orlicz spaces.

Once Fefferman, Stein, and Weiss introduced the classical Hardy spaces $H^{p}$, they became one of the most important function spaces in analysis. Recently, there have been several extensions of classical Hardy spaces. We have the Hardy-Orlicz spaces which are the Orlicz space version of the classical Hardy spaces (see [18], [17], [28], [35]). The Hardy spaces with variable exponents were introduced and studied in [27] and [30]. The atomic decomposition for weighted Hardy spaces with variable exponents is developed in [14].

We also have the atomic decompositions for the Hardy-Morrey spaces (see [11], [16]). The reader may also consult [31] and [37] for some detailed studies on the Triebel-Lizorkin-Morrey spaces which are generalizations of the Hardy-Morrey spaces.

Copyright 2016 by the Tusi Mathematical Research Group.

Received Aug. 24, 2015; Accepted Nov. 11, 2015.

2010 Mathematics Subject Classification. Primary 42B30; Secondary 42B25, 42B35, 46E30.

Keywords. atomic decomposition, molecular decomposition, Hardy spaces, Musielak-Orlicz spaces. 


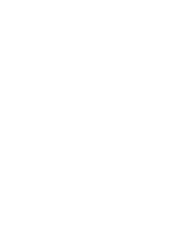

Banach J. Math. Anal. 10 (2016), no. 3, 566-592

http://dx.doi.org/10.1215/17358787-3607354

ISSN: $1735-8787$ (electronic)

http://projecteuclid.org/bjma

\title{
INTRINSIC ATOMIC AND MOLECULAR DECOMPOSITIONS OF HARDY-MUSIELAK-ORLICZ SPACES
}

\author{
KWOK-PUN HO
}

Communicated by T. Sugawa

\begin{abstract}
We introduce the Hardy type space for Musielak-Orlicz spaces. It includes several existing Hardy type spaces such as the Hardy-Orlicz spaces and the Hardy spaces with variable exponents. Furthermore, we develop an atomic decomposition such that the size condition just relies on the norms of Musielak-Orlicz spaces. This gives us a nature extension of the molecular decompositions to the Hardy type space for Musielak-Orlicz spaces.
\end{abstract}

\section{INTRODUCTION}

In this article we aim to establish the atomic and molecular decompositions for the Hardy spaces built on the Musielak-Orlicz spaces.

Once Fefferman, Stein, and Weiss introduced the classical Hardy spaces $H^{p}$, they became one of the most important function spaces in analysis. Recently, there have been several extensions of classical Hardy spaces. We have the Hardy-Orlicz spaces which are the Orlicz space version of the classical Hardy spaces (see [18], [17], [28], [35]). The Hardy spaces with variable exponents were introduced and studied in [27] and [30]. The atomic decomposition for weighted Hardy spaces with variable exponents is developed in [14].

We also have the atomic decompositions for the Hardy-Morrey spaces (see [11], [16]). The reader may also consult [31] and [37] for some detailed studies on the Triebel-Lizorkin-Morrey spaces which are generalizations of the Hardy-Morrey spaces.

Copyright 2016 by the Tusi Mathematical Research Group.

Received Aug. 24, 2015; Accepted Nov. 11, 2015.

2010 Mathematics Subject Classification. Primary 42B30; Secondary 42B25, 42B35, 46E30.

Keywords. atomic decomposition, molecular decomposition, Hardy spaces, Musielak-Orlicz spaces. 


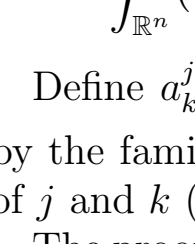

Banach J. Math. Anal. 10 (2016), no. 3, 566-592

http://dx.doi.org/10.1215/17358787-3607354

ISSN: $1735-8787$ (electronic)

http://projecteuclid.org/bjma

\title{
INTRINSIC ATOMIC AND MOLECULAR DECOMPOSITIONS OF HARDY-MUSIELAK-ORLICZ SPACES
}

\author{
KWOK-PUN HO
}

Communicated by T. Sugawa

\begin{abstract}
We introduce the Hardy type space for Musielak-Orlicz spaces. It includes several existing Hardy type spaces such as the Hardy-Orlicz spaces and the Hardy spaces with variable exponents. Furthermore, we develop an atomic decomposition such that the size condition just relies on the norms of Musielak-Orlicz spaces. This gives us a nature extension of the molecular decompositions to the Hardy type space for Musielak-Orlicz spaces.
\end{abstract}

\section{INTRODUCTION}

In this article we aim to establish the atomic and molecular decompositions for the Hardy spaces built on the Musielak-Orlicz spaces.

Once Fefferman, Stein, and Weiss introduced the classical Hardy spaces $H^{p}$, they became one of the most important function spaces in analysis. Recently, there have been several extensions of classical Hardy spaces. We have the Hardy-Orlicz spaces which are the Orlicz space version of the classical Hardy spaces (see [18], [17], [28], [35]). The Hardy spaces with variable exponents were introduced and studied in [27] and [30]. The atomic decomposition for weighted Hardy spaces with variable exponents is developed in [14].

We also have the atomic decompositions for the Hardy-Morrey spaces (see [11], [16]). The reader may also consult [31] and [37] for some detailed studies on the Triebel-Lizorkin-Morrey spaces which are generalizations of the Hardy-Morrey spaces.

Copyright 2016 by the Tusi Mathematical Research Group.

Received Aug. 24, 2015; Accepted Nov. 11, 2015.

2010 Mathematics Subject Classification. Primary 42B30; Secondary 42B25, 42B35, 46E30.

Keywords. atomic decomposition, molecular decomposition, Hardy spaces, Musielak-Orlicz spaces. 


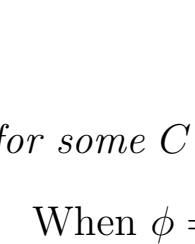

Banach J. Math. Anal. 10 (2016), no. 3, 566-592

http://dx.doi.org/10.1215/17358787-3607354

ISSN: $1735-8787$ (electronic)

http://projecteuclid.org/bjma

\title{
INTRINSIC ATOMIC AND MOLECULAR DECOMPOSITIONS OF HARDY-MUSIELAK-ORLICZ SPACES
}

\author{
KWOK-PUN HO
}

Communicated by T. Sugawa

\begin{abstract}
We introduce the Hardy type space for Musielak-Orlicz spaces. It includes several existing Hardy type spaces such as the Hardy-Orlicz spaces and the Hardy spaces with variable exponents. Furthermore, we develop an atomic decomposition such that the size condition just relies on the norms of Musielak-Orlicz spaces. This gives us a nature extension of the molecular decompositions to the Hardy type space for Musielak-Orlicz spaces.
\end{abstract}

\section{INTRODUCTION}

In this article we aim to establish the atomic and molecular decompositions for the Hardy spaces built on the Musielak-Orlicz spaces.

Once Fefferman, Stein, and Weiss introduced the classical Hardy spaces $H^{p}$, they became one of the most important function spaces in analysis. Recently, there have been several extensions of classical Hardy spaces. We have the Hardy-Orlicz spaces which are the Orlicz space version of the classical Hardy spaces (see [18], [17], [28], [35]). The Hardy spaces with variable exponents were introduced and studied in [27] and [30]. The atomic decomposition for weighted Hardy spaces with variable exponents is developed in [14].

We also have the atomic decompositions for the Hardy-Morrey spaces (see [11], [16]). The reader may also consult [31] and [37] for some detailed studies on the Triebel-Lizorkin-Morrey spaces which are generalizations of the Hardy-Morrey spaces.

Copyright 2016 by the Tusi Mathematical Research Group.

Received Aug. 24, 2015; Accepted Nov. 11, 2015.

2010 Mathematics Subject Classification. Primary 42B30; Secondary 42B25, 42B35, 46E30.

Keywords. atomic decomposition, molecular decomposition, Hardy spaces, Musielak-Orlicz spaces. 


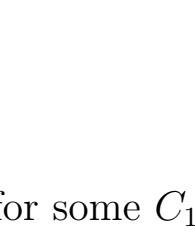

Banach J. Math. Anal. 10 (2016), no. 3, 566-592

http://dx.doi.org/10.1215/17358787-3607354

ISSN: $1735-8787$ (electronic)

http://projecteuclid.org/bjma

\title{
INTRINSIC ATOMIC AND MOLECULAR DECOMPOSITIONS OF HARDY-MUSIELAK-ORLICZ SPACES
}

\author{
KWOK-PUN HO
}

Communicated by T. Sugawa

\begin{abstract}
We introduce the Hardy type space for Musielak-Orlicz spaces. It includes several existing Hardy type spaces such as the Hardy-Orlicz spaces and the Hardy spaces with variable exponents. Furthermore, we develop an atomic decomposition such that the size condition just relies on the norms of Musielak-Orlicz spaces. This gives us a nature extension of the molecular decompositions to the Hardy type space for Musielak-Orlicz spaces.
\end{abstract}

\section{INTRODUCTION}

In this article we aim to establish the atomic and molecular decompositions for the Hardy spaces built on the Musielak-Orlicz spaces.

Once Fefferman, Stein, and Weiss introduced the classical Hardy spaces $H^{p}$, they became one of the most important function spaces in analysis. Recently, there have been several extensions of classical Hardy spaces. We have the Hardy-Orlicz spaces which are the Orlicz space version of the classical Hardy spaces (see [18], [17], [28], [35]). The Hardy spaces with variable exponents were introduced and studied in [27] and [30]. The atomic decomposition for weighted Hardy spaces with variable exponents is developed in [14].

We also have the atomic decompositions for the Hardy-Morrey spaces (see [11], [16]). The reader may also consult [31] and [37] for some detailed studies on the Triebel-Lizorkin-Morrey spaces which are generalizations of the Hardy-Morrey spaces.

Copyright 2016 by the Tusi Mathematical Research Group.

Received Aug. 24, 2015; Accepted Nov. 11, 2015.

2010 Mathematics Subject Classification. Primary 42B30; Secondary 42B25, 42B35, 46E30.

Keywords. atomic decomposition, molecular decomposition, Hardy spaces, Musielak-Orlicz spaces. 


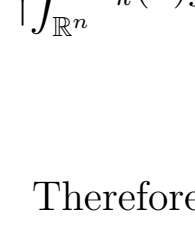

Banach J. Math. Anal. 10 (2016), no. 3, 566-592

http://dx.doi.org/10.1215/17358787-3607354

ISSN: $1735-8787$ (electronic)

http://projecteuclid.org/bjma

\title{
INTRINSIC ATOMIC AND MOLECULAR DECOMPOSITIONS OF HARDY-MUSIELAK-ORLICZ SPACES
}

\author{
KWOK-PUN HO
}

Communicated by T. Sugawa

\begin{abstract}
We introduce the Hardy type space for Musielak-Orlicz spaces. It includes several existing Hardy type spaces such as the Hardy-Orlicz spaces and the Hardy spaces with variable exponents. Furthermore, we develop an atomic decomposition such that the size condition just relies on the norms of Musielak-Orlicz spaces. This gives us a nature extension of the molecular decompositions to the Hardy type space for Musielak-Orlicz spaces.
\end{abstract}

\section{INTRODUCTION}

In this article we aim to establish the atomic and molecular decompositions for the Hardy spaces built on the Musielak-Orlicz spaces.

Once Fefferman, Stein, and Weiss introduced the classical Hardy spaces $H^{p}$, they became one of the most important function spaces in analysis. Recently, there have been several extensions of classical Hardy spaces. We have the Hardy-Orlicz spaces which are the Orlicz space version of the classical Hardy spaces (see [18], [17], [28], [35]). The Hardy spaces with variable exponents were introduced and studied in [27] and [30]. The atomic decomposition for weighted Hardy spaces with variable exponents is developed in [14].

We also have the atomic decompositions for the Hardy-Morrey spaces (see [11], [16]). The reader may also consult [31] and [37] for some detailed studies on the Triebel-Lizorkin-Morrey spaces which are generalizations of the Hardy-Morrey spaces.

Copyright 2016 by the Tusi Mathematical Research Group.

Received Aug. 24, 2015; Accepted Nov. 11, 2015.

2010 Mathematics Subject Classification. Primary 42B30; Secondary 42B25, 42B35, 46E30.

Keywords. atomic decomposition, molecular decomposition, Hardy spaces, Musielak-Orlicz spaces. 


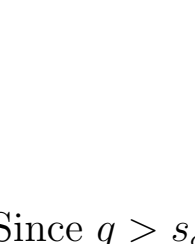

Banach J. Math. Anal. 10 (2016), no. 3, 566-592

http://dx.doi.org/10.1215/17358787-3607354

ISSN: $1735-8787$ (electronic)

http://projecteuclid.org/bjma

\title{
INTRINSIC ATOMIC AND MOLECULAR DECOMPOSITIONS OF HARDY-MUSIELAK-ORLICZ SPACES
}

\author{
KWOK-PUN HO
}

Communicated by T. Sugawa

\begin{abstract}
We introduce the Hardy type space for Musielak-Orlicz spaces. It includes several existing Hardy type spaces such as the Hardy-Orlicz spaces and the Hardy spaces with variable exponents. Furthermore, we develop an atomic decomposition such that the size condition just relies on the norms of Musielak-Orlicz spaces. This gives us a nature extension of the molecular decompositions to the Hardy type space for Musielak-Orlicz spaces.
\end{abstract}

\section{INTRODUCTION}

In this article we aim to establish the atomic and molecular decompositions for the Hardy spaces built on the Musielak-Orlicz spaces.

Once Fefferman, Stein, and Weiss introduced the classical Hardy spaces $H^{p}$, they became one of the most important function spaces in analysis. Recently, there have been several extensions of classical Hardy spaces. We have the Hardy-Orlicz spaces which are the Orlicz space version of the classical Hardy spaces (see [18], [17], [28], [35]). The Hardy spaces with variable exponents were introduced and studied in [27] and [30]. The atomic decomposition for weighted Hardy spaces with variable exponents is developed in [14].

We also have the atomic decompositions for the Hardy-Morrey spaces (see [11], [16]). The reader may also consult [31] and [37] for some detailed studies on the Triebel-Lizorkin-Morrey spaces which are generalizations of the Hardy-Morrey spaces.

Copyright 2016 by the Tusi Mathematical Research Group.

Received Aug. 24, 2015; Accepted Nov. 11, 2015.

2010 Mathematics Subject Classification. Primary 42B30; Secondary 42B25, 42B35, 46E30.

Keywords. atomic decomposition, molecular decomposition, Hardy spaces, Musielak-Orlicz spaces. 


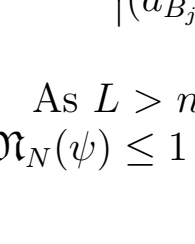

Banach J. Math. Anal. 10 (2016), no. 3, 566-592

http://dx.doi.org/10.1215/17358787-3607354

ISSN: $1735-8787$ (electronic)

http://projecteuclid.org/bjma

\title{
INTRINSIC ATOMIC AND MOLECULAR DECOMPOSITIONS OF HARDY-MUSIELAK-ORLICZ SPACES
}

\author{
KWOK-PUN HO
}

Communicated by T. Sugawa

\begin{abstract}
We introduce the Hardy type space for Musielak-Orlicz spaces. It includes several existing Hardy type spaces such as the Hardy-Orlicz spaces and the Hardy spaces with variable exponents. Furthermore, we develop an atomic decomposition such that the size condition just relies on the norms of Musielak-Orlicz spaces. This gives us a nature extension of the molecular decompositions to the Hardy type space for Musielak-Orlicz spaces.
\end{abstract}

\section{INTRODUCTION}

In this article we aim to establish the atomic and molecular decompositions for the Hardy spaces built on the Musielak-Orlicz spaces.

Once Fefferman, Stein, and Weiss introduced the classical Hardy spaces $H^{p}$, they became one of the most important function spaces in analysis. Recently, there have been several extensions of classical Hardy spaces. We have the Hardy-Orlicz spaces which are the Orlicz space version of the classical Hardy spaces (see [18], [17], [28], [35]). The Hardy spaces with variable exponents were introduced and studied in [27] and [30]. The atomic decomposition for weighted Hardy spaces with variable exponents is developed in [14].

We also have the atomic decompositions for the Hardy-Morrey spaces (see [11], [16]). The reader may also consult [31] and [37] for some detailed studies on the Triebel-Lizorkin-Morrey spaces which are generalizations of the Hardy-Morrey spaces.

Copyright 2016 by the Tusi Mathematical Research Group.

Received Aug. 24, 2015; Accepted Nov. 11, 2015.

2010 Mathematics Subject Classification. Primary 42B30; Secondary 42B25, 42B35, 46E30.

Keywords. atomic decomposition, molecular decomposition, Hardy spaces, Musielak-Orlicz spaces. 


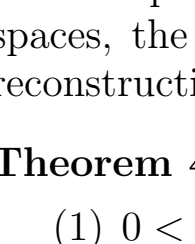

Banach J. Math. Anal. 10 (2016), no. 3, 566-592

http://dx.doi.org/10.1215/17358787-3607354

ISSN: $1735-8787$ (electronic)

http://projecteuclid.org/bjma

\title{
INTRINSIC ATOMIC AND MOLECULAR DECOMPOSITIONS OF HARDY-MUSIELAK-ORLICZ SPACES
}

\author{
KWOK-PUN HO
}

Communicated by T. Sugawa

\begin{abstract}
We introduce the Hardy type space for Musielak-Orlicz spaces. It includes several existing Hardy type spaces such as the Hardy-Orlicz spaces and the Hardy spaces with variable exponents. Furthermore, we develop an atomic decomposition such that the size condition just relies on the norms of Musielak-Orlicz spaces. This gives us a nature extension of the molecular decompositions to the Hardy type space for Musielak-Orlicz spaces.
\end{abstract}

\section{INTRODUCTION}

In this article we aim to establish the atomic and molecular decompositions for the Hardy spaces built on the Musielak-Orlicz spaces.

Once Fefferman, Stein, and Weiss introduced the classical Hardy spaces $H^{p}$, they became one of the most important function spaces in analysis. Recently, there have been several extensions of classical Hardy spaces. We have the Hardy-Orlicz spaces which are the Orlicz space version of the classical Hardy spaces (see [18], [17], [28], [35]). The Hardy spaces with variable exponents were introduced and studied in [27] and [30]. The atomic decomposition for weighted Hardy spaces with variable exponents is developed in [14].

We also have the atomic decompositions for the Hardy-Morrey spaces (see [11], [16]). The reader may also consult [31] and [37] for some detailed studies on the Triebel-Lizorkin-Morrey spaces which are generalizations of the Hardy-Morrey spaces.

Copyright 2016 by the Tusi Mathematical Research Group.

Received Aug. 24, 2015; Accepted Nov. 11, 2015.

2010 Mathematics Subject Classification. Primary 42B30; Secondary 42B25, 42B35, 46E30.

Keywords. atomic decomposition, molecular decomposition, Hardy spaces, Musielak-Orlicz spaces. 


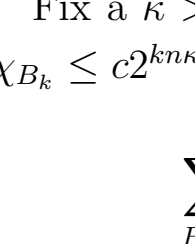

Banach J. Math. Anal. 10 (2016), no. 3, 566-592

http://dx.doi.org/10.1215/17358787-3607354

ISSN: $1735-8787$ (electronic)

http://projecteuclid.org/bjma

\title{
INTRINSIC ATOMIC AND MOLECULAR DECOMPOSITIONS OF HARDY-MUSIELAK-ORLICZ SPACES
}

\author{
KWOK-PUN HO
}

Communicated by T. Sugawa

\begin{abstract}
We introduce the Hardy type space for Musielak-Orlicz spaces. It includes several existing Hardy type spaces such as the Hardy-Orlicz spaces and the Hardy spaces with variable exponents. Furthermore, we develop an atomic decomposition such that the size condition just relies on the norms of Musielak-Orlicz spaces. This gives us a nature extension of the molecular decompositions to the Hardy type space for Musielak-Orlicz spaces.
\end{abstract}

\section{INTRODUCTION}

In this article we aim to establish the atomic and molecular decompositions for the Hardy spaces built on the Musielak-Orlicz spaces.

Once Fefferman, Stein, and Weiss introduced the classical Hardy spaces $H^{p}$, they became one of the most important function spaces in analysis. Recently, there have been several extensions of classical Hardy spaces. We have the Hardy-Orlicz spaces which are the Orlicz space version of the classical Hardy spaces (see [18], [17], [28], [35]). The Hardy spaces with variable exponents were introduced and studied in [27] and [30]. The atomic decomposition for weighted Hardy spaces with variable exponents is developed in [14].

We also have the atomic decompositions for the Hardy-Morrey spaces (see [11], [16]). The reader may also consult [31] and [37] for some detailed studies on the Triebel-Lizorkin-Morrey spaces which are generalizations of the Hardy-Morrey spaces.

Copyright 2016 by the Tusi Mathematical Research Group.

Received Aug. 24, 2015; Accepted Nov. 11, 2015.

2010 Mathematics Subject Classification. Primary 42B30; Secondary 42B25, 42B35, 46E30.

Keywords. atomic decomposition, molecular decomposition, Hardy spaces, Musielak-Orlicz spaces. 


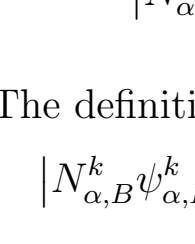

Banach J. Math. Anal. 10 (2016), no. 3, 566-592

http://dx.doi.org/10.1215/17358787-3607354

ISSN: $1735-8787$ (electronic)

http://projecteuclid.org/bjma

\title{
INTRINSIC ATOMIC AND MOLECULAR DECOMPOSITIONS OF HARDY-MUSIELAK-ORLICZ SPACES
}

\author{
KWOK-PUN HO
}

Communicated by T. Sugawa

\begin{abstract}
We introduce the Hardy type space for Musielak-Orlicz spaces. It includes several existing Hardy type spaces such as the Hardy-Orlicz spaces and the Hardy spaces with variable exponents. Furthermore, we develop an atomic decomposition such that the size condition just relies on the norms of Musielak-Orlicz spaces. This gives us a nature extension of the molecular decompositions to the Hardy type space for Musielak-Orlicz spaces.
\end{abstract}

\section{INTRODUCTION}

In this article we aim to establish the atomic and molecular decompositions for the Hardy spaces built on the Musielak-Orlicz spaces.

Once Fefferman, Stein, and Weiss introduced the classical Hardy spaces $H^{p}$, they became one of the most important function spaces in analysis. Recently, there have been several extensions of classical Hardy spaces. We have the Hardy-Orlicz spaces which are the Orlicz space version of the classical Hardy spaces (see [18], [17], [28], [35]). The Hardy spaces with variable exponents were introduced and studied in [27] and [30]. The atomic decomposition for weighted Hardy spaces with variable exponents is developed in [14].

We also have the atomic decompositions for the Hardy-Morrey spaces (see [11], [16]). The reader may also consult [31] and [37] for some detailed studies on the Triebel-Lizorkin-Morrey spaces which are generalizations of the Hardy-Morrey spaces.

Copyright 2016 by the Tusi Mathematical Research Group.

Received Aug. 24, 2015; Accepted Nov. 11, 2015.

2010 Mathematics Subject Classification. Primary 42B30; Secondary 42B25, 42B35, 46E30.

Keywords. atomic decomposition, molecular decomposition, Hardy spaces, Musielak-Orlicz spaces. 


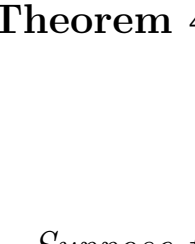

Banach J. Math. Anal. 10 (2016), no. 3, 566-592

http://dx.doi.org/10.1215/17358787-3607354

ISSN: $1735-8787$ (electronic)

http://projecteuclid.org/bjma

\title{
INTRINSIC ATOMIC AND MOLECULAR DECOMPOSITIONS OF HARDY-MUSIELAK-ORLICZ SPACES
}

\author{
KWOK-PUN HO
}

Communicated by T. Sugawa

\begin{abstract}
We introduce the Hardy type space for Musielak-Orlicz spaces. It includes several existing Hardy type spaces such as the Hardy-Orlicz spaces and the Hardy spaces with variable exponents. Furthermore, we develop an atomic decomposition such that the size condition just relies on the norms of Musielak-Orlicz spaces. This gives us a nature extension of the molecular decompositions to the Hardy type space for Musielak-Orlicz spaces.
\end{abstract}

\section{INTRODUCTION}

In this article we aim to establish the atomic and molecular decompositions for the Hardy spaces built on the Musielak-Orlicz spaces.

Once Fefferman, Stein, and Weiss introduced the classical Hardy spaces $H^{p}$, they became one of the most important function spaces in analysis. Recently, there have been several extensions of classical Hardy spaces. We have the Hardy-Orlicz spaces which are the Orlicz space version of the classical Hardy spaces (see [18], [17], [28], [35]). The Hardy spaces with variable exponents were introduced and studied in [27] and [30]. The atomic decomposition for weighted Hardy spaces with variable exponents is developed in [14].

We also have the atomic decompositions for the Hardy-Morrey spaces (see [11], [16]). The reader may also consult [31] and [37] for some detailed studies on the Triebel-Lizorkin-Morrey spaces which are generalizations of the Hardy-Morrey spaces.

Copyright 2016 by the Tusi Mathematical Research Group.

Received Aug. 24, 2015; Accepted Nov. 11, 2015.

2010 Mathematics Subject Classification. Primary 42B30; Secondary 42B25, 42B35, 46E30.

Keywords. atomic decomposition, molecular decomposition, Hardy spaces, Musielak-Orlicz spaces. 


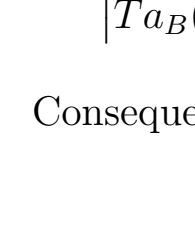

Banach J. Math. Anal. 10 (2016), no. 3, 566-592

http://dx.doi.org/10.1215/17358787-3607354

ISSN: $1735-8787$ (electronic)

http://projecteuclid.org/bjma

\title{
INTRINSIC ATOMIC AND MOLECULAR DECOMPOSITIONS OF HARDY-MUSIELAK-ORLICZ SPACES
}

\author{
KWOK-PUN HO
}

Communicated by T. Sugawa

\begin{abstract}
We introduce the Hardy type space for Musielak-Orlicz spaces. It includes several existing Hardy type spaces such as the Hardy-Orlicz spaces and the Hardy spaces with variable exponents. Furthermore, we develop an atomic decomposition such that the size condition just relies on the norms of Musielak-Orlicz spaces. This gives us a nature extension of the molecular decompositions to the Hardy type space for Musielak-Orlicz spaces.
\end{abstract}

\section{INTRODUCTION}

In this article we aim to establish the atomic and molecular decompositions for the Hardy spaces built on the Musielak-Orlicz spaces.

Once Fefferman, Stein, and Weiss introduced the classical Hardy spaces $H^{p}$, they became one of the most important function spaces in analysis. Recently, there have been several extensions of classical Hardy spaces. We have the Hardy-Orlicz spaces which are the Orlicz space version of the classical Hardy spaces (see [18], [17], [28], [35]). The Hardy spaces with variable exponents were introduced and studied in [27] and [30]. The atomic decomposition for weighted Hardy spaces with variable exponents is developed in [14].

We also have the atomic decompositions for the Hardy-Morrey spaces (see [11], [16]). The reader may also consult [31] and [37] for some detailed studies on the Triebel-Lizorkin-Morrey spaces which are generalizations of the Hardy-Morrey spaces.

Copyright 2016 by the Tusi Mathematical Research Group.

Received Aug. 24, 2015; Accepted Nov. 11, 2015.

2010 Mathematics Subject Classification. Primary 42B30; Secondary 42B25, 42B35, 46E30.

Keywords. atomic decomposition, molecular decomposition, Hardy spaces, Musielak-Orlicz spaces. 


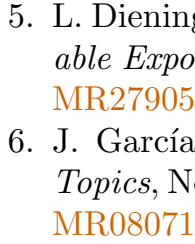

Banach J. Math. Anal. 10 (2016), no. 3, 566-592

http://dx.doi.org/10.1215/17358787-3607354

ISSN: $1735-8787$ (electronic)

http://projecteuclid.org/bjma

\title{
INTRINSIC ATOMIC AND MOLECULAR DECOMPOSITIONS OF HARDY-MUSIELAK-ORLICZ SPACES
}

\author{
KWOK-PUN HO
}

Communicated by T. Sugawa

\begin{abstract}
We introduce the Hardy type space for Musielak-Orlicz spaces. It includes several existing Hardy type spaces such as the Hardy-Orlicz spaces and the Hardy spaces with variable exponents. Furthermore, we develop an atomic decomposition such that the size condition just relies on the norms of Musielak-Orlicz spaces. This gives us a nature extension of the molecular decompositions to the Hardy type space for Musielak-Orlicz spaces.
\end{abstract}

\section{INTRODUCTION}

In this article we aim to establish the atomic and molecular decompositions for the Hardy spaces built on the Musielak-Orlicz spaces.

Once Fefferman, Stein, and Weiss introduced the classical Hardy spaces $H^{p}$, they became one of the most important function spaces in analysis. Recently, there have been several extensions of classical Hardy spaces. We have the Hardy-Orlicz spaces which are the Orlicz space version of the classical Hardy spaces (see [18], [17], [28], [35]). The Hardy spaces with variable exponents were introduced and studied in [27] and [30]. The atomic decomposition for weighted Hardy spaces with variable exponents is developed in [14].

We also have the atomic decompositions for the Hardy-Morrey spaces (see [11], [16]). The reader may also consult [31] and [37] for some detailed studies on the Triebel-Lizorkin-Morrey spaces which are generalizations of the Hardy-Morrey spaces.

Copyright 2016 by the Tusi Mathematical Research Group.

Received Aug. 24, 2015; Accepted Nov. 11, 2015.

2010 Mathematics Subject Classification. Primary 42B30; Secondary 42B25, 42B35, 46E30.

Keywords. atomic decomposition, molecular decomposition, Hardy spaces, Musielak-Orlicz spaces. 


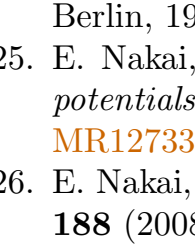

Banach J. Math. Anal. 10 (2016), no. 3, 566-592

http://dx.doi.org/10.1215/17358787-3607354

ISSN: $1735-8787$ (electronic)

http://projecteuclid.org/bjma

\title{
INTRINSIC ATOMIC AND MOLECULAR DECOMPOSITIONS OF HARDY-MUSIELAK-ORLICZ SPACES
}

\author{
KWOK-PUN HO
}

Communicated by T. Sugawa

\begin{abstract}
We introduce the Hardy type space for Musielak-Orlicz spaces. It includes several existing Hardy type spaces such as the Hardy-Orlicz spaces and the Hardy spaces with variable exponents. Furthermore, we develop an atomic decomposition such that the size condition just relies on the norms of Musielak-Orlicz spaces. This gives us a nature extension of the molecular decompositions to the Hardy type space for Musielak-Orlicz spaces.
\end{abstract}

\section{INTRODUCTION}

In this article we aim to establish the atomic and molecular decompositions for the Hardy spaces built on the Musielak-Orlicz spaces.

Once Fefferman, Stein, and Weiss introduced the classical Hardy spaces $H^{p}$, they became one of the most important function spaces in analysis. Recently, there have been several extensions of classical Hardy spaces. We have the Hardy-Orlicz spaces which are the Orlicz space version of the classical Hardy spaces (see [18], [17], [28], [35]). The Hardy spaces with variable exponents were introduced and studied in [27] and [30]. The atomic decomposition for weighted Hardy spaces with variable exponents is developed in [14].

We also have the atomic decompositions for the Hardy-Morrey spaces (see [11], [16]). The reader may also consult [31] and [37] for some detailed studies on the Triebel-Lizorkin-Morrey spaces which are generalizations of the Hardy-Morrey spaces.

Copyright 2016 by the Tusi Mathematical Research Group.

Received Aug. 24, 2015; Accepted Nov. 11, 2015.

2010 Mathematics Subject Classification. Primary 42B30; Secondary 42B25, 42B35, 46E30.

Keywords. atomic decomposition, molecular decomposition, Hardy spaces, Musielak-Orlicz spaces. 